DESY 99-028

ISSN 0418-9833

LUNFD6/(NFFL-7167) 1999

\title{
The role of resolved virtual photons in the production of forward jets at HERA
}

\author{
H. Jung, L. Jönsson, H. Küster \\ Department of Physics, \\ Lund University, 22100 Lund, Sweden \\ E-mail: jung@mail.desy.de,leif@quark.lu.se
}

\begin{abstract}
The measurement of forward jet cross sections has been suggested as a promising probe of new small $x$ parton dynamics and the question is whether the new HERA data provide an indication of this. In this paper the influence of resolved photon processes has been investigated and it has been studied to what extent the inclusion of such processes in addition to normal deep inelastic scattering leads to agreement with data. It is shown that two DGLAP evolution chains from the hard scattering process towards the proton and the photon respectively, are sufficient to describe effects observed in the HERA data, which have been attributed to BFKL dynamics.
\end{abstract}

\section{Introduction}

Experimental data from deep inelastic scattering (DIS) in a kinematic region where new parton dynamics is expected to become noticeable, i.e. at small values of the scaled proton momentum, $x$, are not described by models based on interactions with pointlike photons. In a previous paper [1] we have demonstrated that the addition of interactions through resolved photons offers a possible explanation of the observed discrepancies and leads to good agreement with all available data.

This paper is devoted to a more detailed discussion of the resolved photon concept and comparisons with data on forward jet production in DIS, since the forward jet cross section has been advocated as a particularly sensitive measure of small $x$ parton dynamics [2, 3]. Analytic calculations based on the BFKL equation [4, 5] in the leading logarithmic approximation (LLA) are in fair agreement with data. However, recent calculations of the BFKL kernel in the next-to-leading logarithmic approximation (NLLA) [6] have given surprisingly large corrections, and it remains to be shown whether the data can still be reasonably described.

Monte Carlo generators based on direct, point-like photon interactions (DIR model), calculated from leading order (order $\alpha_{s}$ ) QCD matrix elements, and leading log parton 
showers based on the DGLAP evolution do not take any new parton dynamics in the small $x$ region into account and are therefore not expected to fit the experimental data. Recent results from the H1 [7] and ZEUS [8] experiments on forward jet production exhibit significant deviations from the predictions of such models. Also, next-to-leading order calculations (NLO, i.e. order $\alpha_{s}^{2}$ ) assuming point-like photons predict too small a cross section compared to data.

The study of forward jet production with contributions from direct as well as resolved photon processes has been performed using the RAPGAP 2.06 [9, 10] Monte Carlo event generator.

\section{Resolved Photons in DIS}

In electron-proton scattering the internal structure of the proton as well as of the exchanged photon can be resolved provided the scale of the hard subprocess is larger than the inverse radius of the proton, $1 / R_{p}^{2} \sim \Lambda_{Q C D}^{2}$, and the photon, $1 / R_{\gamma}^{2} \sim Q^{2}$, respectively. Resolved photon processes play an important role in photo-production of high $p_{T}$ jets, where $Q^{2} \approx 0$, but they can also give considerable contributions to DIS processes [11, 112] if the scale $\mu^{2}$ of the hard subprocess is larger than $Q^{2}$, the inverse size of the photon. This led to the idea of including contributions from resolved photon processes as part of deep inelastic scattering to get a more complete description [13].

In the following we give a brief description of the model for resolved virtual photons used in the Monte Carlo generator RAPGAP. Given the fractional momentum transfer of the incoming electron to the exchanged photon, the Equivalent Photon Approximation provides the flux of virtual transversely polarized photons [10, 9, and references therein]. The contribution from longitudinally polarized photons has been neglected. The partonic structure of the virtual photon is defined by parameterizations of the parton densities,

$x_{\gamma} f_{\gamma}\left(x_{\gamma}, \mu^{2}, Q^{2}\right)$, which depend on the two scales $\mu^{2}$ and $Q^{2}$ 14, 15, 16,. The following hard subprocesses are considered (RES model): $g g \rightarrow q \bar{q}, g g \rightarrow g g, q g \rightarrow q g, q \bar{q} \rightarrow g g$, $q \bar{q} \rightarrow q \bar{q}, q q \rightarrow q q$. Parton showers on both the proton and the photon side are included. The generic diagram for the process $q_{\gamma} g_{p} \rightarrow q g$ including parton showers is shown in Fig. 1.

Since the photon structure function depends on the scale, $\mu^{2}$, of the hard scattering process, the cross section of resolved photon processes will consequently also depend on the choice of this scale. It has to be carefully considered in which range of $\mu^{2} / Q_{0}^{2}$ the photon-parton cross section can be factorised into a parton-parton cross section convoluted with the parton density of the photon. The parton density of the photon is evolved from a starting scale $Q_{0}^{2}$ to the scale $\mu^{2}$, the virtuality at the hard subprocess, giving a resummation to all orders.

\subsection{Parton Distribution Functions}

Due to factorization of the cross section, the parton densities of both the virtual photon and the proton enter into the calculations. The proton structure function, $F_{2}$, has been measured to high accuracy and therefore the various parameterizations only give marginal 


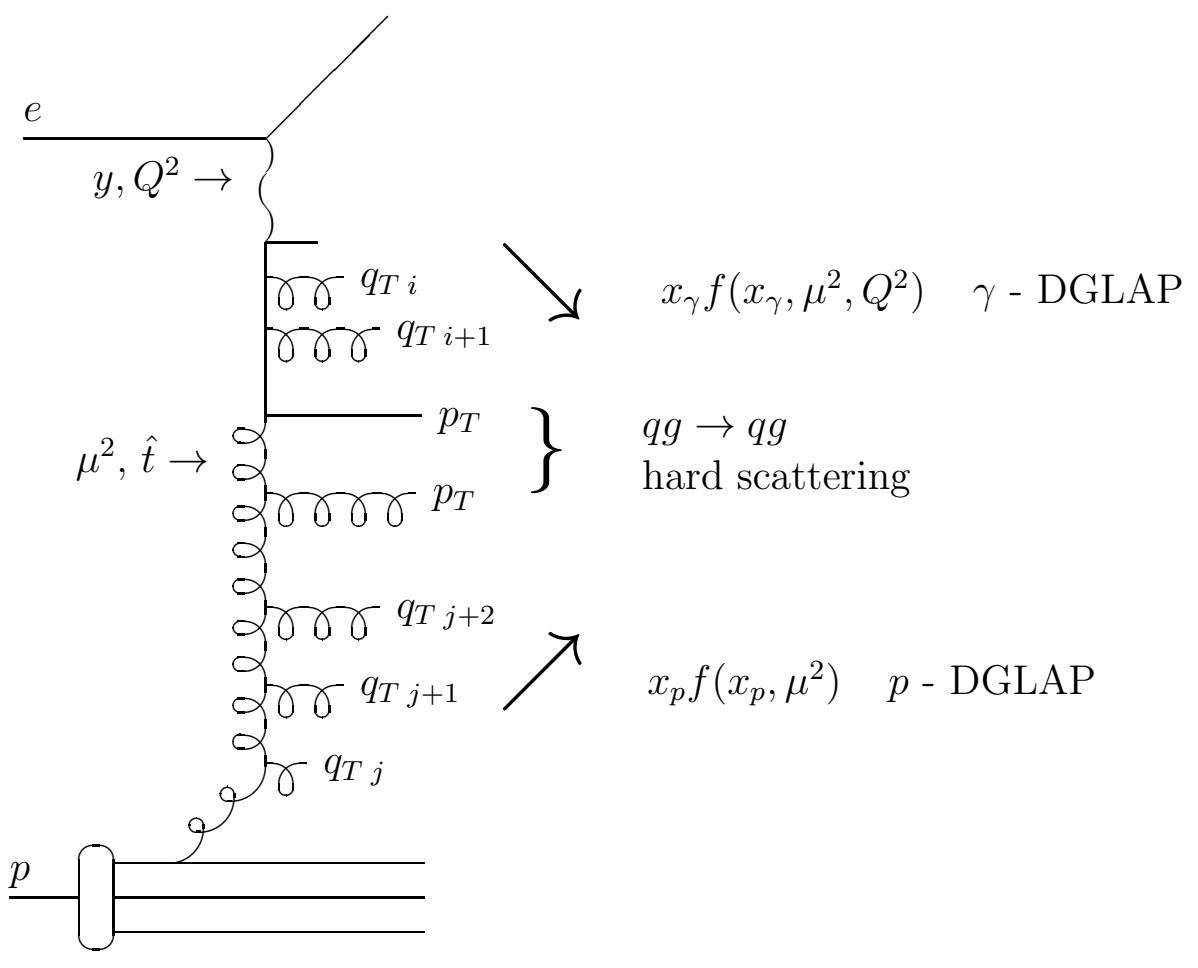

Figure 1: Deep inelastic scattering with a resolved virtual photon and the $q_{\gamma} g_{p} \rightarrow q g$ partonic subprocess.

differences in the measurable kinematic region. Two parameterizations of the parton distribution in the proton, GRV 94 HO (DIS) and CTEQ4D, have been considered, which both give good agreement with the proton structure function data [17, 18]. It was found that the results produced were identical at the percent level, when keeping $\Lambda_{Q C D}$ fixed. In the following we use only GRV HO.

The photon can interact via its partons either in a bound vector meson state or as decoupled partons if the $p_{T}$ of the partons is high enough. The splitting $\gamma \rightarrow q \bar{q}$ is called the anomalous component of the photon. The structure function of virtual photons has been measured only recently, but not nearly to the same precision as the proton structure function. However, it turns out that data are in good agreement with the parameterization of Schuler and Sjöstrand (SaS) 15. The SaS parameterization offers a choice of $Q_{0}^{2}$ values at which the anomalous part becomes effective. We have studied these choices resulting in different magnitudes of the parton densities, and consequently of the cross sections. For the SaS parameterization we have used $Q_{0}^{2}$ as given by eq.(12) of ref. [15] $(I P 2=2)$. This choice is also suitable for a description of other hadronic final state properties (not considered in this paper), like energy flow, forward particle spectra and jet cross sections.

The hadronic contribution to the virtual photon structure function decreases rapidly with increasing $Q^{2}$, which means that the main contribution at large $Q^{2}$ comes from the anomalous piece in the photon splitting. This is completely calculable in pQCD and leads to an expected agreement between the parameterization of Glück - Reya - Stratman [14] 
and that of Schuler - Sjöstrand [15], but also with the simple ansatz of Drees - Godbole 16]. However differences exist in the way the hadronic part of the structure function is matched to the pointlike part, which just reflects the theoretical uncertainty.

In this study we will restrict ourselves to the SaS parton distributions [15].

\subsection{Choice of Scale}

In leading order $\alpha_{s}$ processes, the renormalization scale $\mu_{R}$ and factorization scale $\mu_{F}$ are not well defined, which allows a number of reasonable choices. There are essentially two competing effects: a large scale suppresses $\alpha_{s}\left(\mu^{2}\right)$ but gives, on the other hand, an increased parton density, $x f\left(x, \mu^{2}\right)$, for a fixed small $x$ value. The net effect depends on the details of the interaction and on the parton density parameterization.

In previous papers [19, 1] we have tried different scales like $\mu^{2}=4 \cdot p_{T}^{2}$ and $\mu^{2}=Q^{2}+p_{T}^{2}$, and found that these choices gave similar results.

However, in resolved virtual photon processes, the choice of the scale $\mu^{2}$, at which the photon is probed, is severely restricted [20]. In a partonic process $a+b \rightarrow c+d$, where $a, b, c, d$ denote four-vectors and where parton $a$ has the virtuality $Q^{2}$, the transverse momentum $p_{T}^{2}$ of parton $c$ is given in the small angle limit $(-\hat{t} \ll \hat{s})$ by: $p_{T}^{2}=\hat{s}(-\hat{t}) /(\hat{s}+$ $Q^{2}$ ), with $\hat{s}$ and $\hat{t}$ being the usual Mandelstam variables. In a $t$ channel process the virtuality is given by $\mu^{2}=-\hat{t}$. Thus we have:?

$$
\mu^{2}=-\hat{t}=p_{T}^{2}+Q^{2} \cdot \frac{p_{T}^{2}}{\hat{s}}<Q^{2}+p_{T}^{2}
$$

From eq.(1) we see that the scale $\mu^{2}$ is always larger than the transverse momentum squared of the hard partons and less than $Q^{2}+p_{T}^{2}$. In the following we will use $\mu^{2}=Q^{2}+p_{T}^{2}$ as scale for both resolved virtual photon processes and for direct photon processes. This choice of scale provides a smooth transition from the kinematic region of normal DIS into the range where resolved photons start contributing and further into the photo-production region. The same scale has also been used in NLO calculations including resolved photons in deep inelastic scattering [21, 22].

A basic test that the scale is reasonable is that the parton shower evolution scheme should not be able to produce partons with transverse momenta larger than those produced by the matrix element for the hard scattering process.

\section{Forward Jets}

HERA has extended the available $x$ region down to values below $10^{-4}$, where new parton dynamics might show up. Based on calculations in the LLA of the BFKL kernel, the cross section for DIS events at low $x$ and large $Q^{2}$ with a high $p_{T}^{2}$ jet in the proton direction (a forward jet) [2, 3] is expected to rise more rapidly with decreasing $x$ than expected from DGLAP based calculations. New results from the H1 [7] and ZEUS [8] experiments have recently been published. The data can be described neither by conventional DIR Monte

\footnotetext{
${ }^{1}$ We are grateful to T. Sjöstrand for pointing out this simple explanation
} 
Carlo models nor by a NLO calculation, while comparisons to analytic calculations of the LLA BFKL mechanism has proven reasonable agreement.

It should be kept in mind that both the NLO calculations and the BFKL based calculations are performed on the parton level whereas the data are at the level of hadrons.

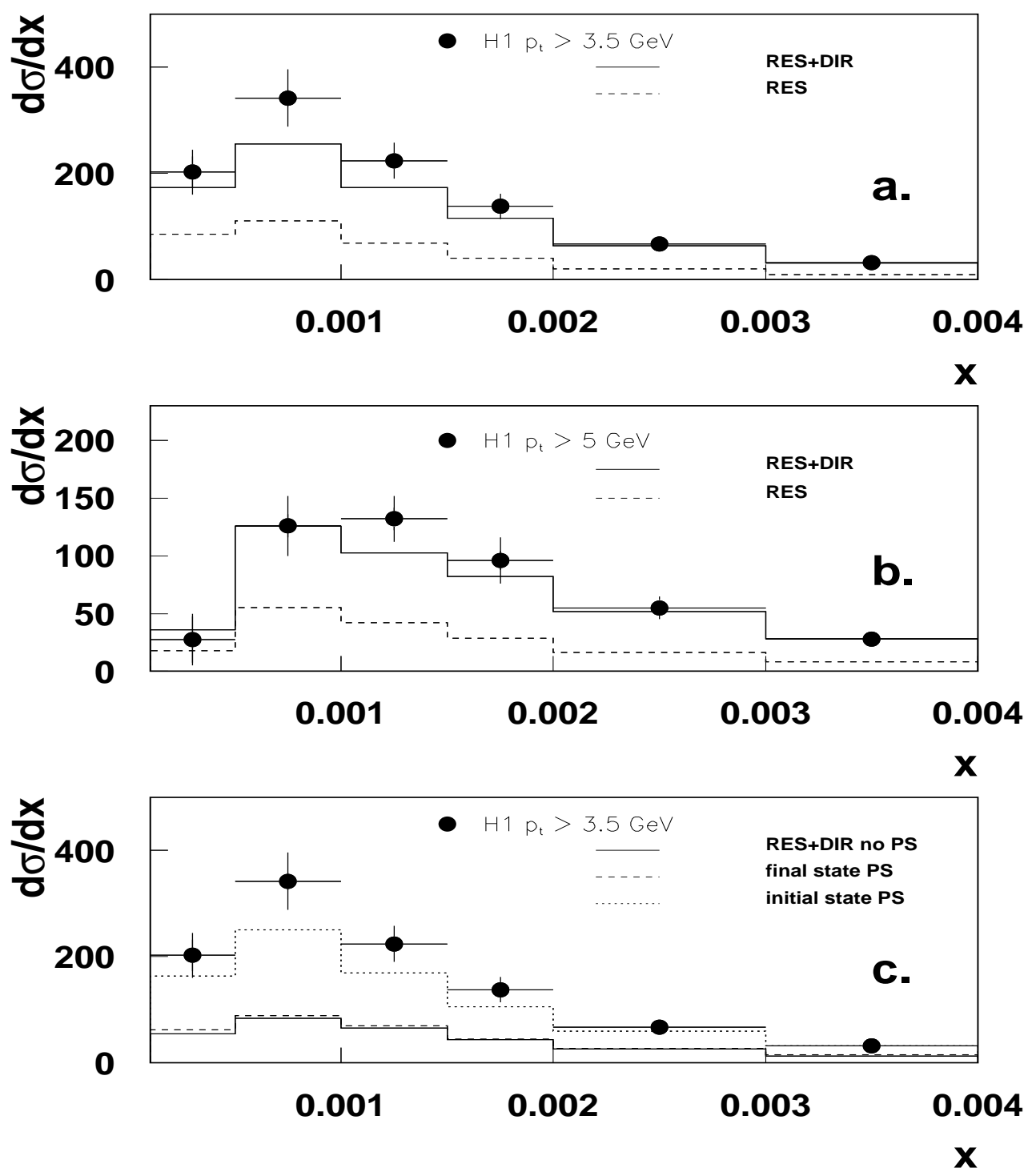

Figure 2: The forward jet cross section as a function of $x$ for $p_{T \text { jet }}>3.5 \mathrm{GeV}$ (a) and $p_{T \text { jet }}>5 \mathrm{GeV}(b)$ as measured by H1 [7] . Also shown are the RAPGAP predictions for the sum of direct and resolved processes (solid line) as well as the resolved photon contribution alone (dashed line). The RAPGAP predictions for the sum of direct and resolved processes without initial and final state parton showers (solid line), including only final state (dashed line) and only initial state parton showers (dotted line) are shown in $(c)$. 
In Fig. 2 $2 a$ and $b$ the forward jet cross section as measured by the H1 collaboration [7 is compared to the prediction of the RAPGAP Monte Carlo generator for both resolved photon process alone (labeled RES) and for the sum of direct and resolved processes (labeled DIR+RES). The calculation is performed with a scale $\mu^{2}=Q^{2}+p_{T}^{2}$ used in the parton densities and in the determination of $\alpha_{s}$ for both direct and resolved photon processes. Only if both processes are included, is the Monte Carlo able to give a good description of the measurements. The forward jet cross section as measured by the ZEUS experiment [8] can be equally well described by this Monte Carlo program using the same structure functions and parameter setting.

Recently a full NLO calculation of the forward jet cross section was performed [22] with contributions from both direct and resolved virtual photons. Comparisons with H1 and ZEUS data exhibit good agreement while it is found that LO calculations fall below the experimental data. The assumption is that the parton showers in the RAPGAP model account for higher order effects, which would explain the good description of data from this model.

As a consequence, the influence of the initial and final state QCD cascade on the forward jet cross section has been studied in more detail and compared to the calculations. In Fig. $2 c$ we show the contribution to the forward jet cross section of H1 [7] coming from pure matrix element calculations and the effect of including initial and final state radiation. It is observed that the final state radiation gives essentially no additional contribution to the matrix element cross section whereas the initial state radiation plays an important role in bringing the Monte Carlo predictions to into agreement with data. We, thus, come to the same conclusion as in [22], that the LO order DIR+RES matrix elements are not enough to describe the measured forward jet cross section. However, with initial and final state parton showers included, the data are described well and it seems that the parton showers are able to simulate contributions from higher order processes. We also find that the LO cross section calculations of [22] agree well with the RAPGAP results in LO order, i.e. when parton showers are switched off. It should be noted that the contribution from the direct process with initial and final state parton showers included, corresponding to the difference between the RES+DIR and RES histograms in Fig. 2 2 , is too small to give agreement with data.

It is also important to make sure that the hardness of the scale doesn't lead to parton cascades which produce radiation with larger $p_{T}$ than produced by the hard scattering matrix element. In Fig. 3 the ratio of the transverse momentum of any initial state parton $q_{T}^{2}$ to the transverse momentum $p_{T}^{2}$ of the hard scattering process is shown in the $\gamma^{*} p$ CMS for events which satisfy the forward jet analysis criteria. The solid line corresponds to $\mu^{2}=Q^{2}+p_{T}^{2}$, the dotted line to $\mu^{2}=4 \cdot p_{T}^{2}$ and the dashed line to $\mu^{2}=p_{T}^{2}$. One can see that essentially all partons coming from the initial state cascade have transverse momenta smaller than the partons of the hard scattering $p_{T}$, which is expected in a DGLAP type evolution, where the transverse momenta are ordered in $q_{T}$ towards the hard scattering process. Thus we conclude that the scale $\mu^{2}=Q^{2}+p_{T}^{2}$ which has been used for this study fullfils the requirements of a DGLAP type initial state cascade.

In Fig. 4 the different contributions of the total resolved photon cross section are shown separately within the cuts of the forward jet analysis. From Fig. Ga it is observed that the hadronic part of the virtual photon structure function, as expected, gives a negligible 


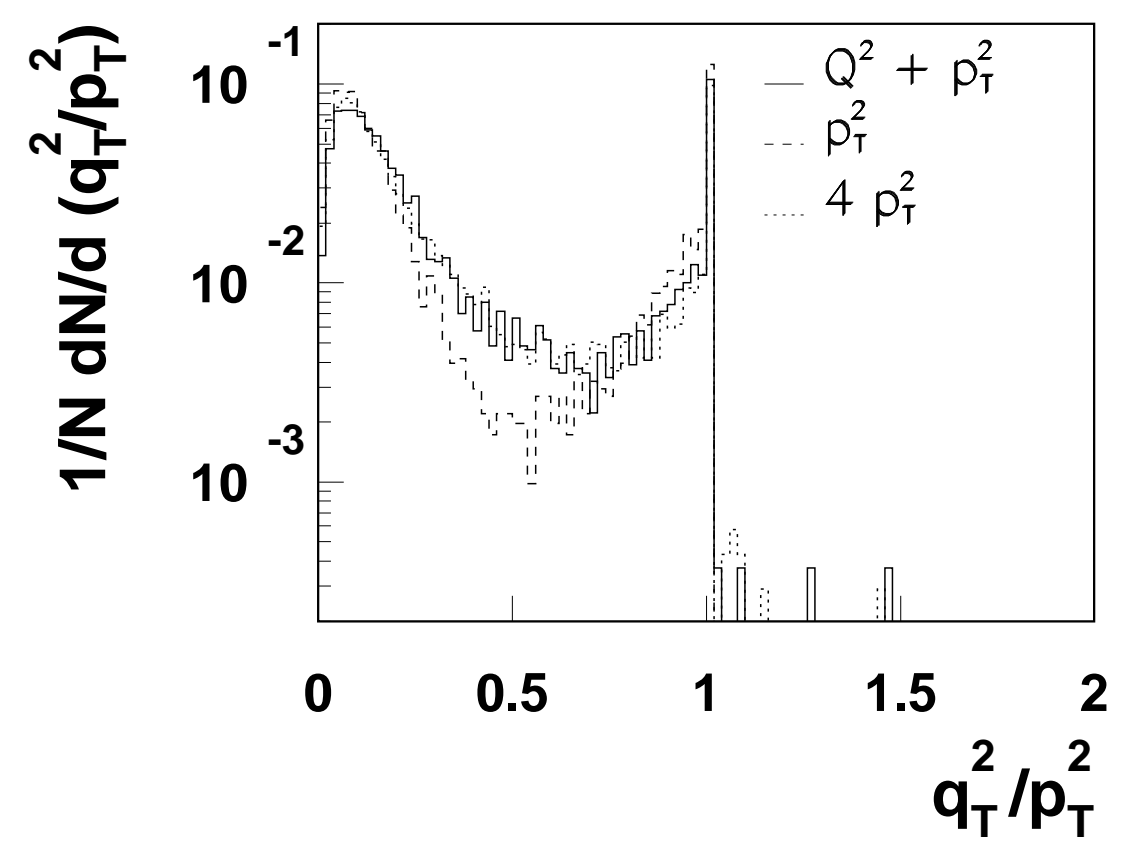

Figure 3: The ratio $R=q_{T}^{2} / p_{T}^{2}$ of the transverse momenta $q_{T}^{2}$ of partons from the initial state cascade to the transverse momentum $p_{T}^{2}$ of the partons from the hard scattering process. The solid line corresponds to the scale $\mu^{2}=Q^{2}+p_{T}^{2}$, the dotted line to $\mu^{2}=4 \cdot P_{T}^{2}$ and the dashed line to $\mu^{2}=p_{T}^{2}$. The distribution is normalized to the total number of entries. Please note the logarithmic scale on the $y$-axis.

contribution to the measured cross section, since it dies off rapidly with increasing $Q^{2}$. Fig. $₫ b$ shows that the subprocess $q_{\gamma} g_{p} \rightarrow q g$ contributes the most to the resolved photon cross section in the forward jet region $(\sim 60 \%)$ and that the subprocesses $q q \rightarrow q q$, $q \bar{q} \rightarrow q \bar{q}$ and $g g \rightarrow g g$ each give a contribution of the order of $10 \%$.

A small fraction of the DIS events, fulfilling the selection criteria for forward jets, actually contains two identified jets. Analytic calculations (in LLA) 23] have been performed in the same kinematic region and with the same jet selection as defined for the H1 one-jet sample. The predicted ratio varies from $3 \%$ to $6 \%$ as $x$ increases from $0.5 \cdot 10^{-3}$ to $x=3 \cdot 10^{-3}$. Our previously reported prediction from the RAPGAP generator [1] including both direct and resolved photon processes was that about $1 \%$ of the total forward jet sample contains two forward jets. This is about a factor of 3 lower than the 

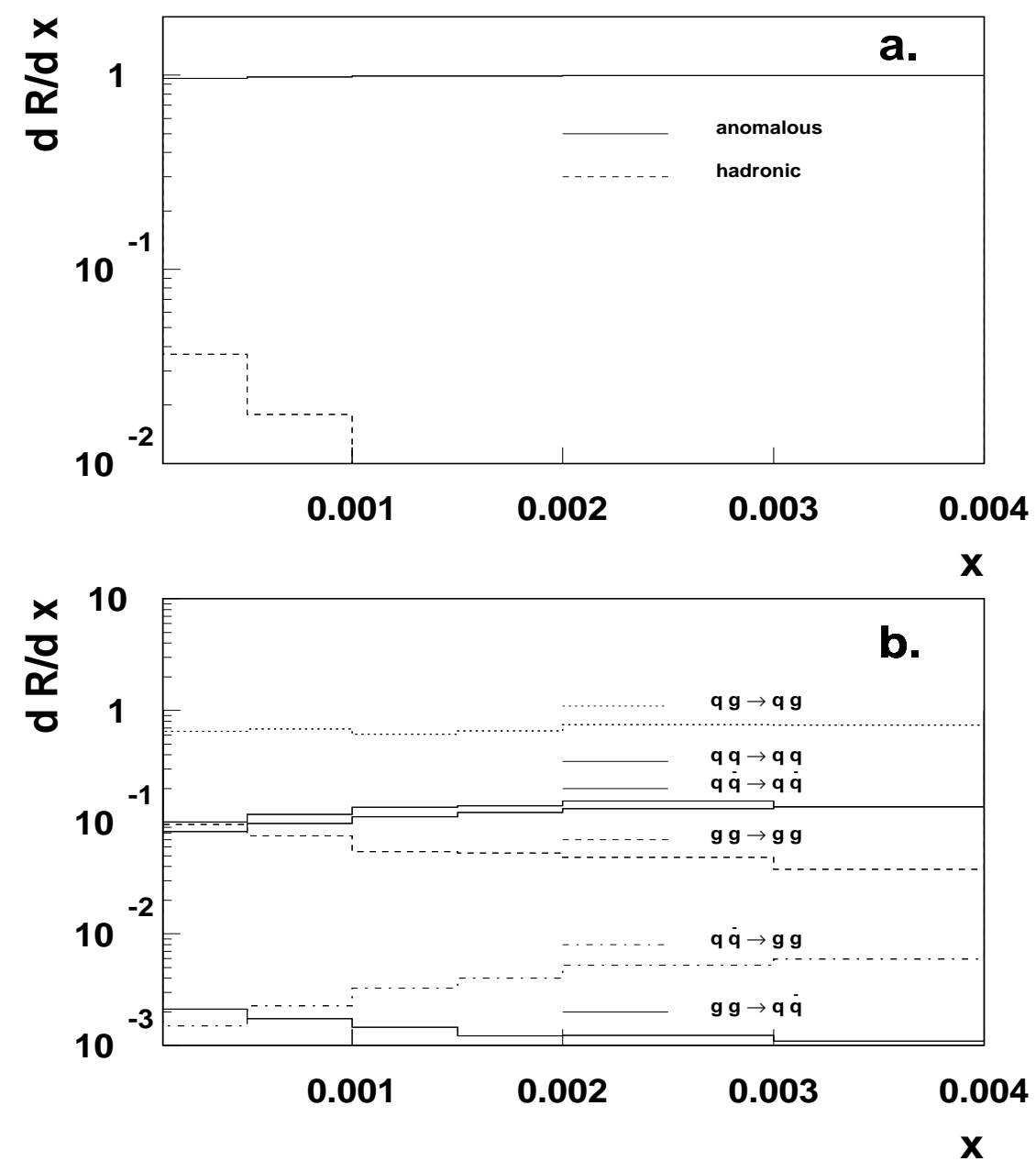

Figure 4: Different contributions to the total cross section of resolved virtual photons within the cuts of the forward jet analysis [7]. In $(a)$ is shown the ratio $R=\frac{\sigma_{i}}{\sigma_{\text {res. tot }}}$, i.e. the anomalous (pointlike) part (solid line) and the hadronic part (dashed line), respectively, of the resolved virtual photon cross section divided by the the total resolved photon cross section as a function of $x$. In $(b)$ is shown the ratio $R=\frac{\sigma_{i}}{\sigma_{\text {res. } t o t}}$ as a function of $x$ for different subprocesses $i: q g \rightarrow q g$ (dotted line), $q q \rightarrow q q$ and $q \bar{q} \rightarrow q \bar{q}$ (upper solid line), $g g \rightarrow g g$ (dashed line), $q \bar{q} \rightarrow g g$ (dashed-dotted line) and $g g \rightarrow q \bar{q}$ (lower solid line).

prediction from the BFKL calculations but a large part of this discrepancy could be due to hadronization effects which would reduce the prediction of the parton level BFKL calculation. Recently this ratio has been measured by the $\mathrm{H} 1$ experiment [7] to be $1 \%$, in excellent agreement with the prediction of RAPGAP. This gives further confidence in the 
basic concept of resolved photons even at large $Q^{2}$.

The ZEUS collaboration has presented a measurement of the forward jet cross section as a function of $E_{T}^{2} / Q^{2}$ [24] in the kinematic region $Q^{2}>10 \mathrm{GeV}^{2}, y>0.1, E_{e^{\prime}}>10$ $\mathrm{GeV}, \eta_{\text {jet }}<2.6, x_{\text {jet }}>0.036, E_{T \text { jet }}>5 \mathrm{GeV}, p_{z \text { breit }}>0 \mathrm{GeV}, 2.5 \cdot 10^{-4}<x<8 \cdot 10^{-2}$ but without implementing the DGLAP suppression cut $0.5<E_{T}^{2} / Q^{2}<2$. The results were compared to the predictions from different Monte Carlo programs and the conclusion is that only the RAPGAP DIS generator including interactions through resolved virtual photons can describe the data over the full range in $E_{T}^{2} / Q^{2}$. In Fig. $5 a$ the ZEUS results are shown together with the prediction of RAPGAP and in Fig. $5 b$ the contributions coming from the hadronic and the pointlike part of the virtual photon structure function are presented separately. The influence of initial and final state radiation is shown in Fig. 5c which again illustrates that the initial state radiation gives an essential contribution to the cross section while the contribution from final state radiation is negligible.

\section{Summary and Discussion}

Recent experimental data on forward jet production show deviations from traditional LO Monte Carlo models assuming directly interacting point-like photons. It is tempting to assume that the observed effects could be explained by BFKL dynamics.

In the present study we have shown that the addition of resolved photon processes to the direct interactions in DIS leads to good agreement with the data. This agreement does not depend on any specific choice of scale or tuning of any other parameters in the RAPGAP generator. The best evidence of the universality of this approach is that, with the same parameter setting, it is possible to describe a wide range of other data like the transverse energy flow [25], transverse momentum spectra of single particles [26], the $(2+1)$ jet rate [27] and single inclusive jet cross sections [11], as we have shown in [1].

We have observed that the dominant contributions to the resolved photon processes come from order $\alpha_{s}^{2}$ diagrams with the hard subprocess $q_{\gamma} g_{p} \rightarrow q g$ (see Fig. 11). Since the partons which form the photon remnant per definition have smaller $p_{T}$ than the partons involved in the hard scattering, a situation with non $q_{T}$ ordering is created.

In the LO DIR model, the ladder of gluon emissions is governed by DGLAP dynamics giving a strong ordering of $q_{T}$ for emissions between the photon and the proton vertex. The models describing resolved photon processes and BFKL dynamics are similar in the sense that both lead to a breaking of this ordering in $q_{T}$. The BFKL picture, however, allows for complete dis-ordering in $q_{T}$, while in the resolved photon case the DGLAP ladder is split into two shorter ladders, one from the hard subsystem to the proton vertex and one to the photon vertex, each of them ordered in $q_{t}$ (see Fig. 1). Only if the ladders are long enough to produce additional hard radiation might it be possible to separate resolved photon processes from processes governed by BFKL dynamics. Thus the resolved photon approach may be a "sufficiently good" approximation to an exact BFKL calculation and the two approaches may prove indistinguishable within the range of $x$ accessible at HERA.

It should be emphasized again that the usual NLO calculation assuming point-like virtual photons contains a significant part of what is attributed to the resolved structure of the virtual photon in the LO scheme. [21]. The NLO calculations including contribu- 
tions from direct as well as resolved photons, however, are similar to using LO matrix elements for both the photon and the proton with the addition of parton showers, as implemented in the RAPGAP generator. Recent calculations have proven good agreement with the predictions of RAPGAP and thus also with data. This indicates that higher order contributions are well simulated by the inclusion of parton showers in LO Monte Carlo generators.

\section{Acknowledgments}

It is a pleasure to thank G. Ingelman and A. Edin for discussions about the concept of resolved photons. We have also profited from a continuous dialogue with B. Andersson, G. Gustafson and T. Sjöstrand. We want to thank G. Kramer and B. Pötter for many discussions on the relation between resolved photons in DIS and its relation to NLO calculations. We also want to thank M. Wüsthoff and J. Bartels for discussions on BFKL and the resolved photons in DIS.

\section{References}

[1] H. Jung, L. Jönsson, H. Küster, Resolved Photon processes in DIS and Small $x$ Dynamics, DESY 98-051 and hep-ph/9805396.

[2] A. Mueller, Nucl. Phys. B (Proc. Suppl) 18C (1990) 125.

[3] A. Mueller, J. Phys. G 17 (1991) 1443.

[4] V. Fadin, E. Kuraev, L. Lipatov, Sov. Phys. JETP 45 (1977) 199.

[5] Y. Balitskii, L. Lipatov, Sov. J. Nucl. Phys. 28 (1978) 822.

[6] V. Fadin, L. Lipatov, Phys. Lett. B 429 (1998) 127, hep-ph/9802290.

[7] H1 Collaboration, C. Adloff et al., Nucl. Phys. B (1998), DESY 98-143.

[8] ZEUS Collaboration; J. Breitweg et al., Eur. Phys. J. (1998), DESY 98-050.

[9] H. Jung, Comp. Phys. Comm. 86 (1995) 147.

[10] H. Jung, The RAPGAP Monte Carlo for Deep Inelastic Scattering, version 2.06, Lund University, 1998, http://www-h1.desy.de/ jung/rapgap.html.

[11] H1 Collaboration, C. Adloff et al., Phys. Lett. B 415 (1997) 418, DESY-97-179.

[12] J. Chyla, J. Cvach, Virtual Photon Structure from jet production at HERA, in Proc. of the Workshop on Future Physics at HERA, edited by A. De Roeck, G. Ingelman, R. Klanner (Hamburg, 1996).

[13] A. Edin, G. Ingelman, private discussion, 1996. 
[14] M. Glück, E. Reya, M. Stratman, Phys. Rev. D 54 (1996) 5515.

[15] G. Schuler, T. Sjöstrand, Phys. Lett. B 376 (1996) 193.

[16] M. Drees, R. Godbole, Phys. Rev. D 50 (1994) 3124.

[17] H1 Collaboration; C. Adloff et al., Nucl. Phys. B 497 (1997) 3, hep-ex/9703012.

[18] ZEUS Collaboration; J. Breitweg et al., Phys. Lett. B 407 (1997) 432, DESY 97-135.

[19] H. Jung, Resolved Photons and BFKL - Type Signatures in DIS, in Proc. of the Madrid low $x$ workshop, edited by F. Barreiro (Miraflores de la Sierra, Spain, 1997), hep-ph/9709425.

[20] G. Gustafson, T. Sjöstrand, private communications, 1997.

[21] G. Kramer, B. Potter, Eur. Phys. J. C 5 (1998) 261, hep-ph/9804352.

[22] G. Kramer, B. Poetter, , DESY 99-004 and hep-ph/9901314.

[23] J. Kwiecinski, C. A. M. Lewis, A. D. Martin, Phys. Rev. D57 (1998) 496, hepph/9707375.

[24] ZEUS Collaboration; J. Breitweg et al., Addendum to: Forward Jet Production in Deep Inelastic Scattering at HERA, Contributed paper to ICHEP98, Abstract 804, Vancover, 1998.

[25] H1 Collaboration; I. Abt et al., Z. Phys. C 63 (1994) 377, DESY 94-033.

[26] H1 Collaboration, C. Adloff et al., Nucl. Phys. B 485 (1997) 3, DESY-96-215.

[27] H1 Collaboration, T. Ahmed et al., , DESY 98-076 and hep-ex/9806029. 

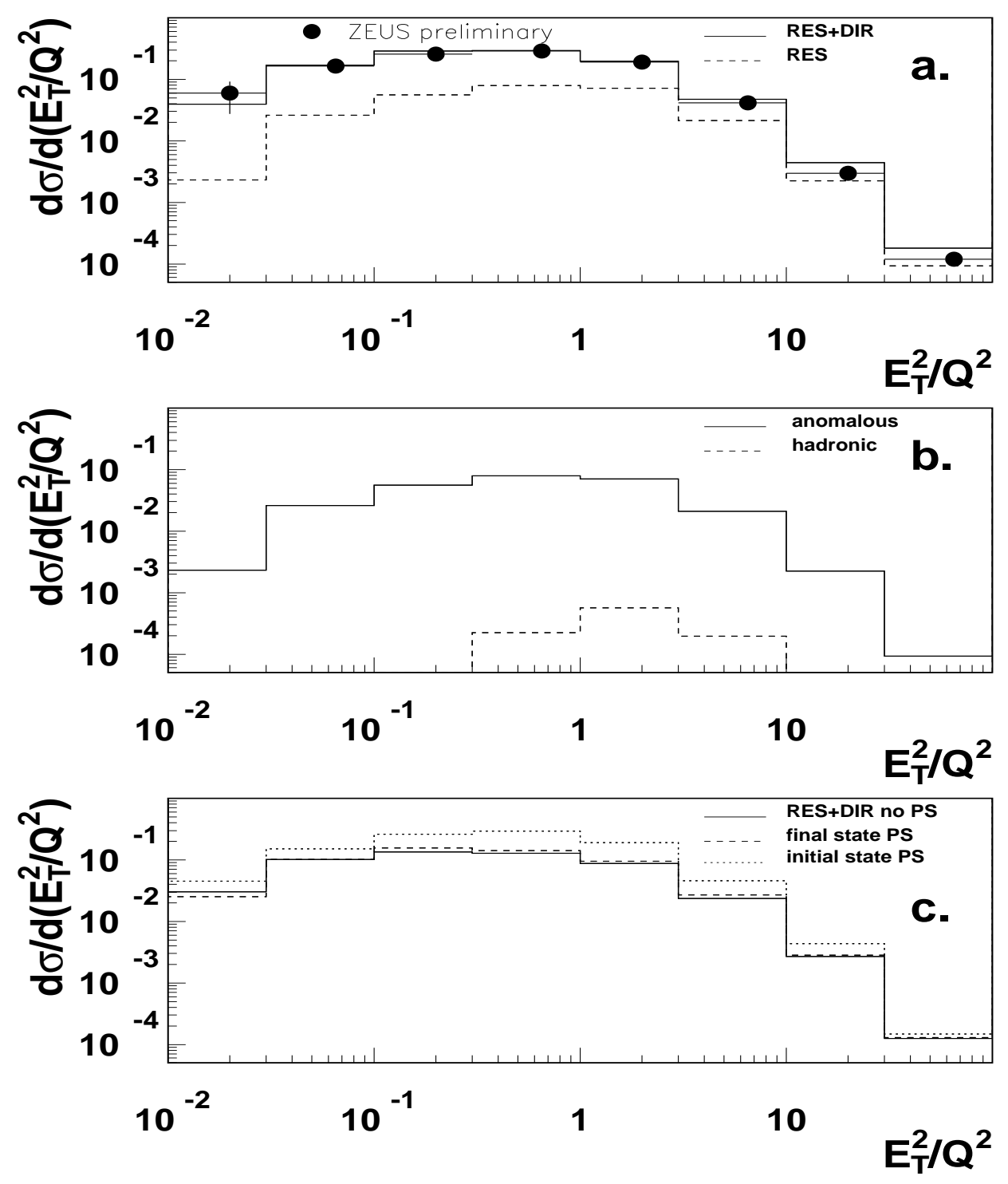

Figure 5: The cross section of forward jets as a function of $E_{T}^{2} / Q^{2}$ as measured by ZEUS [24]. In $(a)$ the data are compared to the prediction of RAPGAP. The solid line shows the sum of direct and resolved virtual photon contributions, whereas the dashed line shows the resolved photon contribution alone. In $(b)$ is shown the part of the cross section coming from the anomalous component (solid line) and the one coming from the hadronic component of the virtual photon separately. In $(c)$ the RAPGAP predictions are shown for the sum of direct and resolved processes without initial and final state parton showers (solid line), including only final state (dashed line) and only initial state parton showers (dotted line). 